\title{
Subaru/HDS Studies of r-Process Elements in Metal-Poor Stars from Near-UV Spectra
}

\author{
S. Honda ${ }^{1}$, W. Aoki ${ }^{1}$, Y. Ishimaru ${ }^{2}$, S. Wanajo ${ }^{3}$, S. G. Ryan ${ }^{4}$, \\ T. Kajino ${ }^{1}$, H. Ando ${ }^{1}$, and T. C. Beers ${ }^{5}$ \\ ${ }^{1}$ National Astronomical Observatory of Japan, 2-21-1 Osawa, Mitaka, Tokyo, 181-8588, Japan \\ email: honda@optik.mtk.nao.ac.jp \\ ${ }^{2}$ Department of General Education, Kogakuin University, 2665-1 Nakano-Cho, Hachioji, Tokyo \\ 192-0015, Japan \\ ${ }^{3}$ Graduate School of Science, University of Tokyo, Bunkyo-ku, Tokyo 113-0033, Japan \\ ${ }^{4}$ Department of Physics and Astronomy, Open University, Walton Hall, Milton Keynes \\ MK76AA, UK \\ ${ }^{5}$ Department of Physics and Astronomy and JINA: Joint Institute for Nuclear Astrophysics, \\ Michigan State University, East Lansing, MI 48824, USA.
}

\begin{abstract}
We present detailed abundance measurements of neutron-capture elements for the two very metal-poor stars HD 6268 and HD 122563, based on very high-quality, near-UV spectra (S/N >140@3100A) using Subaru/HDS. Abundances have been obtained for a total of 26 and 19 neutron-capture elements in these two stars, respectively, including $\mathrm{Nb}, \mathrm{Mo}, \mathrm{Ru}, \mathrm{Pd}, \mathrm{Ag}$, $\mathrm{Pr}$, and $\mathrm{Sm}$. We have confirmed that the abundance pattern of neutron-capture elements in HD 6268 agrees very well with that of previously known r-process-enhanced stars. In contrast, the elemental abundances of HD 122563 are found to steeply decrease with increasing atomic number than those of HD 6268, and are much lower than than the r-process pattern in solarsystem material. This result provides a new, strong constraint on models of the nucleosynthetic process that has provided light neutron-capture elements in the very early Galaxy.
\end{abstract}

Keywords. Stars: abundances, ultraviolet: stars, nuclear reactions, nucleosynthesis, abundances

\section{Introduction}

Essentially all elements heavier than the iron peak are synthesized by neutron-capture processes. About half of the neutron-capture elements in the solar system are thought to have been provided by the r-process. However, details of the nuclear reactions and the astrophysical site(s) of the r-process are still unclear. An effective method to investigate the nature of r-process is to measure the chemical abundances of very metal-poor stars, in which the heavy elements are considered to have been provided by only a few supernovae. Their surface chemical composition would well preserve the results of individual nucleosynthesis processes.

One remarkable example is CS 22892-052, which exhibits large excesses of r-process elements relative to iron (Sneden et al. 2003). An important result of this and studies of other similar stars (e.g., Hill et al. 2002, Christlieb et al. 2004, Honda et al. 2004) is the discovery of the "universality" of the r-process. The abundance pattern of heavy neutroncapture elements $(56 \leqslant Z \leqslant 76)$ in r-process-enhanced, metal-poor stars is in excellent agreement with that of the r-process component in the solar-system. This fact has had a large impact on the modeling of r-process nucleosynthesis (e.g., Otsuki et al. 2003). However, these studies also revealed that the abundance patterns of light neutron-capture elements $(38 \leqslant Z \leqslant 48)$ are not in agreement with that of the solar system r-process. 


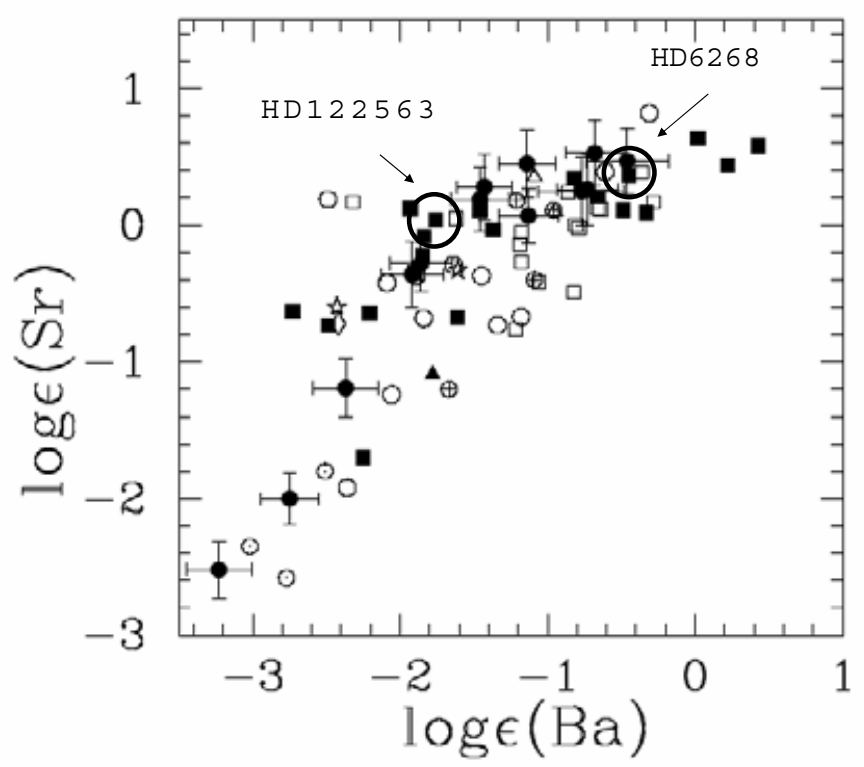

Figure 1. Sr abundances as a function of the Ba abundance for very metal-poor stars with $[\mathrm{Fe} / \mathrm{H}] \leqslant-2.5$ from McWilliam 1998; Burris et al 2000; Carretta et al. 2002; Depagne et al. 2002; Johnson \& Bolte 2002; Ishimaru et al. 2004; Cohen et al. 2004; Honda et al. 2004, and Aoki et al. 2005. Stars having significant excesses of carbon and s-process elements are excluded. The targets discussed in the present work are labelled.

\section{Previous observations and sample selection}

We have observed 40 very metal-poor stars using the High Dispersion Spectrograph (HDS, Noguchi et al. 2002) of the Subaru Telescope, and determined the abundances of numerous neutron-capture elements (Honda et al. 2004, Aoki et al. 2005). We confirmed that the abundance patterns of the heavy neutron-capture elements $(56 \leqslant Z \leqslant 70)$ in seven objects with excesses of these elements are similar to that of the solar system rprocess component. However, the light neutron-capture elements $(38 \leqslant Z \leqslant 47$ ) exhibit large deviations from the solar pattern.

We adopt $\operatorname{Sr}(Z=38)$ and $\mathrm{Ba}(Z=56)$ as representatives of the light and heavy neutron-capture elements, respectively. Figure 1 shows the correlation between $\mathrm{Sr}$ and Ba abundances in very metal-poor stars. This figure shows that (1) there is no Sr-poor star with high Ba abundance, and that (2) the dispersion of $\mathrm{Sr}$ abundance is remarkable in Bapoor stars. This trend can be explained by hypothesizing following two nucleosynthesis processes. One is a process which produces $\mathrm{Sr}$ and Ba with similar proportions. The other is a process producing Sr without Ba. While the former is known as the "main" r-process associated with the production of heavy neutron-capture elements, the latter process has been observationally and theoretically studied only quite recently (e.g., Burris et al. 2000; Wasserburg \& Qian 2000; Truran et al. 2002; Travaglio et al. 2004).

In order to further investigate the two likely processes, we selected two bright metalpoor stars which are thought to be representative examples of the two processes: HD 6268 exhibits overabundances of heavy neutron-capture elements such as Ba, while HD 122563 shows high abundances of light neutron-capture elements with no excess of heavier ones. 


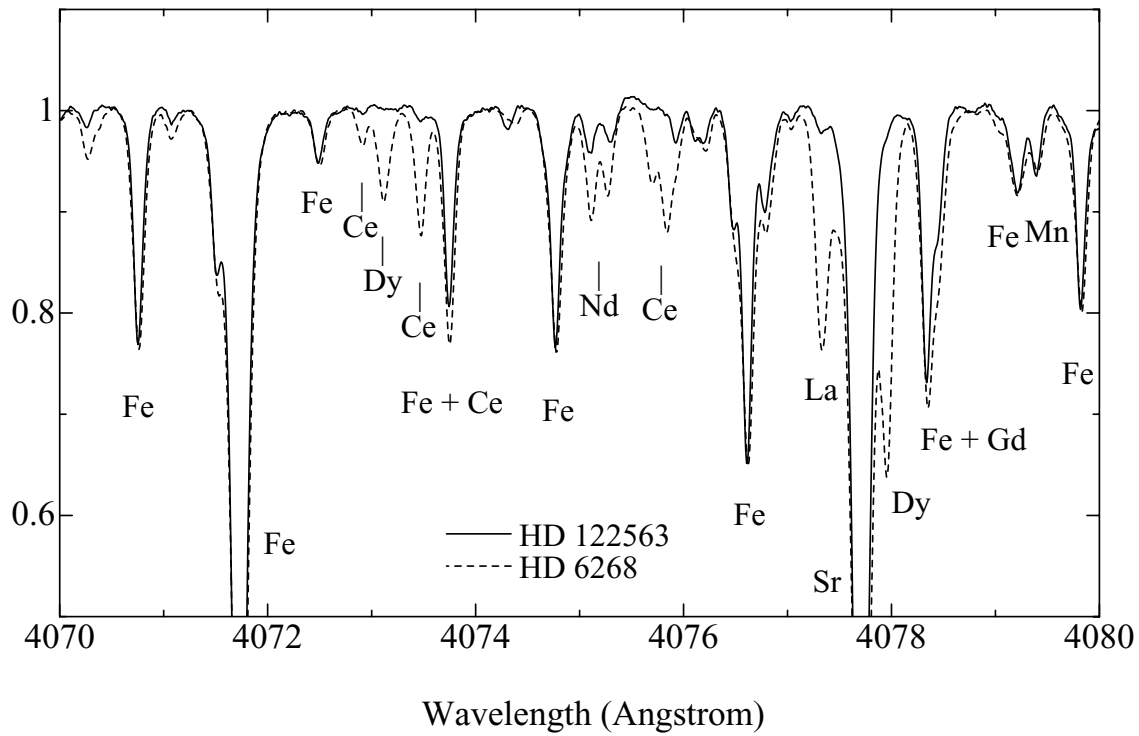

Figure 2. Comparison of a red portion of the spectra for HD 6268 (dashed line) and HD 122563 (solid line).

\section{Near-UV observations with Subaru/HDS}

High-resolution, near-UV spectroscopy $(3000-4000 \AA)$ was obtained for these two stars with Subaru/HDS in order to measure the absorption lines of neutron-capture elements. Although our targets are bright stars, a large telescope is required because near-UV observations with ground-based telescopes is made difficult due to strong absorption by the Earth's atmosphere. In order to maximize our ability to detect as many lines as possible in this crowded spectral region a high resolving power $(R=90,000)$ was chosen. The signal-to-noise ratios $(\mathrm{S} / \mathrm{N})$ of the spectra are $350 / 1$ at $3500 \AA ; 700 / 1$ at $4000 \AA$ for HD 6268, and 480/1 at $3500 \AA$; and $900 / 1$ at $4000 \AA$ for HD 122563 .

Figure 2 shows a comparison of a red portion of the spectra of the two stars. The absorption features of iron and manganese are very similar in these spectra, indicating the atmospheric parameters of HD 6268 and HD 122563 are very similar $\left(T_{\text {eff }}=4600 K\right.$, $\log g=1.0, v_{\text {micro }}=2.1 \mathrm{~km} \mathrm{~s}^{-1}$, and $[\mathrm{Fe} / \mathrm{H}]=-2.63$ for $\mathrm{HD} 6268 ; T_{\text {eff }}=4570 K, \log g=$ $1.1, v_{\text {micro }}=2.2 \mathrm{~km} \mathrm{~s}^{-1}$, and $[\mathrm{Fe} / \mathrm{H}]=-2.77$ for HD 122563). However, those of the neutron-capture elements (e.g., La, Dy, Ce) are clearly much stronger in HD 6268 than in HD 122563.

In contrast, the strengths of the Sr line are quite similar in these two stars, confirming an excess of $\mathrm{Sr}$ in HD 122563 as well as in HD 6268. In order to investigate the abundance patterns between heavy and light neutron-capture elements, we searched for features of elements with $38 \leqslant Z \leqslant 47$. We have detected several light neutron-capture elements (e.g., Nb, Mo, Ru, Pd, Ag, etc.) which have not been observed by many previous studies.

\section{Abundance Patterns of HD 6268 and HD 122563}

We adopted the model atmosphere parameters derived by Honda et al. (2004) for conducting our abundance analyses. The derived abundance patterns are shown in Figure 3. We have detected 26 elements from $\operatorname{Sr}(Z=38)$ to $\operatorname{Th}(Z=90)$ in the spectrum of 

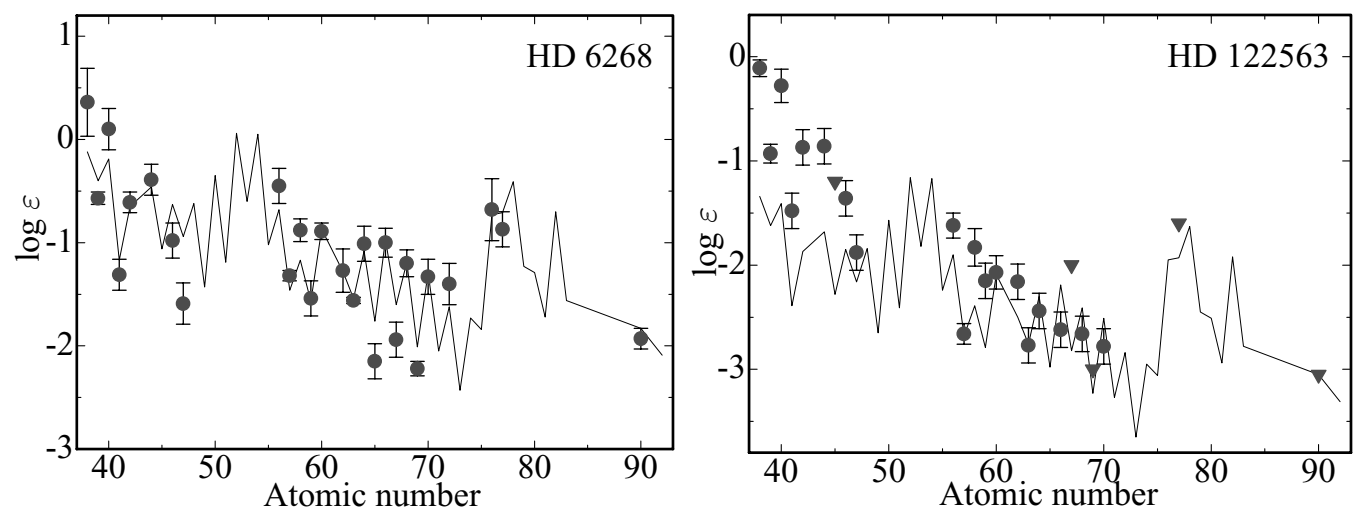

Figure 3. Abundance patterns of HD 6268 and HD 122563. The line is the solar system r-process abundance pattern (Burris et al. 2000), scaled to fit the measured Eu abundance. The triangles represent upper limits for several elements.

HD 6268. We confirmed that the abundance pattern of heavy neutron-capture elements in HD 6268 shows good agreement with the solar-system-r-process pattern, while that of the light ones shows several deviations. We should also note that some departure is found at odd elements ( $\mathrm{Tm}, \mathrm{Ho}, \mathrm{Tb}$ ). In addition, abundance ratios between $\mathrm{Th}$ and other heavy neutron-capture elements (e.g., Eu) of this object is slightly higher than CS 22892-052. These should be investigated in more detail in future work.

Although heavy neutron-capture elements are deficient in HD 122563, we have detected 11 elements with $56 \leqslant Z \leqslant 70$ from our high-quality spectrum. In An additional eight elements with $38 \leqslant Z \leqslant 47$ are also detected. HD 122563 exhibits a significantly different abundance pattern from that of the solar system r-process component; the abundances of light neutron-capture elements are much higher than those of the heavier ones.

In order to display these behaviors more clearly, Figure 4 shows the difference of the abundances between these stars and the solar system r-process pattern, as a function of atomic number. The abundance pattern of HD 6268 are basically in agreement with the solar system r-process pattern, although the agreement is not as good as that of CS 22892052 found by Sneden et al. (2003), and other previously observed r-processed-enhanced, metal-poor stars.

In contrast, our new measurements for HD 122563 clearly demonstrate that the elemental abundances steeply decrease with increasing atomic number, in particular as one moves from the elements near the first abundance peak of the r-process $(Z \leqslant 40)$ to those near the second peak $(\leqslant 56)$. This decreasing trend in the region of $38 \leqslant Z \leqslant 47$ provides an important constraint on modeling the nucleosynthesis process responsible for the light neutron-capture elements (see below).

\section{What is the source of the light neutron-capture elements in HD 122563 ?}

The abundance pattern produced by the "main" r-process can be represented by the elements observed in HD 6268, which have a similar distribution the abundance patterns of many previously studied r-process-enhanced, metal-poor stars. The ratios between light neutron-capture elements and the heavier ones in HD 122563 are clearly very different from those in HD 6268, which indicates that the "main" r-process is not an important source of the light neutron-capture elements in HD 122563. 

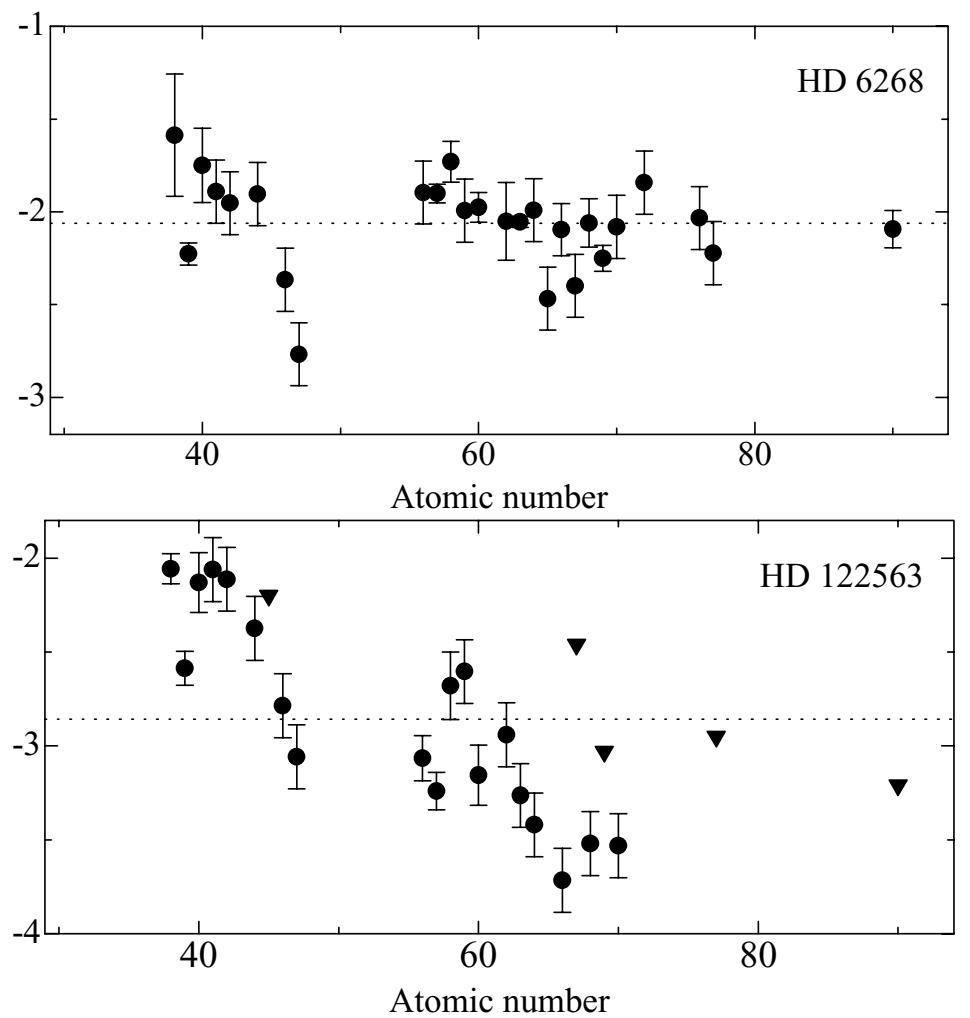

Figure 4. Logarithmic differences from the solar system r-process pattern of HD 6268 and HD 122563. The triangles represent upper limits.

The main s-process is also excluded from the possible source of light neutron-capture elements in HD 122563, because this process is expected to yield predominantly heavy neutron-capture elements (Ba-Pb) compared to light ones (Sr-Zr) (e.g. Busso et al. 1999; Aoki et al. 2002).

The weak component of the s-process has been introduced in order to account for the abundances of the light s-process nuclei in the Solar System. However, the gradually decreasing trend of elemental abundances from Sr to Ba found in HD 122563 does not agree with the that of the weak s-process, which predicts a rapid decrease of abundances at $A \sim 90$ (e.g., Y or Zr). It should be noted that the weak s-process is also expected to be ineffective at low metallicity, because of the lack of neutron sources such as ${ }^{22} \mathrm{Ne}$.

Thus, another process that has efficiently produced light neutron-capture elements at low metallicity is required to explain the abundance pattern of neutron-capture elements in HD 122563. The elemental abundances of HD 122563 provide a new constraint on this process. Recently, the operation of a "weak" r-process has been discussed as one possibility (e.g., Wanajo et al., this volume).

\section{Conclusions}

We have obtained the abundance patterns, covering a wide range of atomic numbers, for the two very metal-poor stars HD 6268 and HD 122563, concentrating on the large number of neutron-capture elements detectable in the near-UV region. Our abundance study revealed that HD 122563 has a significantly different abundance pattern from that 
of HD 6268, which exhibits a similar abundance pattern to previously known r-processrich stars. The elemental abundances of HD 122563 more steeply decrease with increasing atomic number than those of HD 6268, and also with respect to the r-process component in solar-system material. Further theoretical and observational studies to understand the process that is responsible for the light neutron-capture elements in HD 122563, as well as in other halo stars, are strongly desired.

\section{Acknowledgements}

This study is based on data collected at the Subaru Telescope, which is operated by the National Astronomical Observatory of Japan. T.C.B. acknowledges support from grants AST 00-98508, AST 00-98549, AST 04-06784, and PHY 02-16783, Physics Frontier Centers/JINA: Joint Institute for Nuclear Astrophysics, awarded by the US National Science Foundation.

\section{References}

Aoki, W., et al. 2005, ApJ in press

Aoki, W., Norris, J.E., Ryan, S.G., Beers, T.C., \& Ando, H. 2002, ApJ, 567, 1166

Burris, D.L., Pilachowski, C.A., Armandroff, T.A., Sneden, C., Cowan, J.J., \& Roe, H. 2000, ApJ 544, 302

Busso, M., et al. 1999, ARA\&A 37, 239

Carretta, E., Gratton, R., Cohen, J.G., Beers, T.C., \& Christlieb, N. 2002, AJ 124, 481

Christlieb, N., Beers, T.C., Barklem, P.S., Bessell, M., Hill, V. et al., 2004, A $\mathscr{E} A$ 428, 1027

Cohen, J.G., Christlieb, N., McWilliam, A., Shectman, S., Thompson, I. et al., 2004, ApJ 612, 110

Depagne, E., Hill, V., Spite, M., Spite, F., Plez, B. et al., 2002, A\&A 390, 187

Hill, V., Plez, B., Cayrel, R., Beers, T.C., Nordström, B., et al. 2002, A\&A A 387, 560

Honda, S., Aoki, W., Kajino, T., Ando, H., Beers, T.C., et al. 2004, ApJ 607, 474

Ishimaru, Y., Wanajo, S., Aoki, W., \& Ryan, S.G., 2004, ApJ 600, L47

Johnson, J.A. \& Bolte, M. 2002, ApJ 579, 616

McWilliam, A. 1998, AJ 115, 1640

Noguchi, K., Aoki, W., Kawanomoto, S., Ando, H., Honda, S., et al. 2002, PASJ 54, 855

Otsuki, K., Mathews, G., \& Kajino, T., 2003, NewA 8, 767

Sneden, C., Cowan, J., Lawler, J., Ivans, I., Burles, S., et al. 2003, ApJ 591, 936

Travaglio, C., Gallino, R., Arnone, E., Cowan, J., Jordan, F., \& Sneden, C. 2004, ApJ 601, 864

Truran, J.W., Cowan, J., Pilachowski, C., \& Sneden, C., 2002, PASP 114, 1293

Wanajo, S., et al. 2005, This Volume

Wasserburg, G.J. \& Qian, Y.-Z. 2000, ApJ 529, L21 